\title{
The Problem of Setting Traffic Signal Cycles at Crossroads
}

\author{
Dimitra Alexiou $^{1}$, Leonidas Bakouros ${ }^{2}$ \\ ${ }^{1}$ Department of Spatial Planning and Development, School of Engineering, Aristotle University of Thessaloniki, Thssaloniki, Greece \\ ${ }^{2}$ Department of Civil and Environmental Engineering, Imperial College London, London, UK
}

Email address:

dimitraalexiou@plandevel.auth.gr (D. Alexiou), leonidas.bakouros20@imperial.ac.uk (L. Bakouros)

\section{To cite this article:}

Dimitra Alexiou, Leonidas Bakouros. The Problem of Setting Traffic Signal Cycles at Crossroads. American Journal of Applied Mathematics. Vol. 9, No. 3, 2021, pp. 70-74. doi: 10.11648/j.ajam.20210903.12

Received: April 17, 2021; Accepted: May 13, 2021; Published: June 7, 2021

\begin{abstract}
In this paper, the problem of setting traffic light cycles at crossroads and intersections is considered in order to reduce traffic congestion by minimizing total vehicle waiting time. A method to determine the family $\&$ of all discrete cycle phasing systems with the minimum number of phases is presented. The aim is to detect the most appropriate phasing sequence for traffic control corresponding to a current traffic situation from among all the components of $\&$. The method is applied at a complex multi-cross intersection. The problem, dealing with traffic movements and the conflicting relations that arise, is stated within the framework of graph theory. There are several methods for setting traffic signal cycles at traffic light intersections. In this paper and in the context of graph theory, we develop a method which aims to determine the $\wp^{\circ}$ family of all discrete phases of phase systems with the smallest number of phases. The aim is to select from the elements of the $\delta^{\circ}$ the appropriate phase system that corresponds to the current traffic situation.
\end{abstract}

Keywords: Traffic Signal Cycles, Graph Coloring, Pedestrian Crosswalk

\section{Introduction}

Generally, these cycles should consist of the smallest number of phases. In this paper, we present a method for determining the $\wp$ family of all discrete system phase cycles with the smallest number of phases. The aim is to locate $\wp$ among all cycles and to select the optimal phase system that corresponds to the current traffic situation taking into account the activation of pedestrian crossings. The method presented here is applied to a typical traffic junction as well as to an area consisting of several junctions that are directly connected to each other and is called a multi-junction.

The above problem deals with the traffic movements and the incompatible relations among them and is treated in the context of graph theory [4-6].

The design of a circular phase system aims at defining a set of movements that can be active simultaneously (green indicator). Such a system is a phase. A period consists of phases that are activated sequentially and cyclically. When one phase is activated the movements of all other phases are waiting at the red indicator. Also, every movement must be included in at least one phase of the period.

\section{Preliminaries}

A distinct L-coloring of the $\mathrm{G}$ in Conflict graph corresponds to a specific component of the

$$
\wp=\left\{\mathrm{C}_{1}, \mathrm{C}_{2}, \ldots \mathrm{C}_{\mathrm{n}}\right\}
$$

family where each $\mathrm{C}_{1}$ cycle contains $\mathrm{L}$ phases, i.e.

$$
\mathrm{C}_{1}=\left\{\varphi_{\mathrm{i}}^{1}, \varphi_{\mathrm{i}}^{2}, \ldots \varphi_{\mathrm{i}}^{\mathrm{L}}\right\}
$$

The elements of each phase

$$
\varphi_{\mathrm{i}}^{\mathrm{j}}=\left\{\mathrm{m}_{\mathrm{i} 1}^{\mathrm{j}}, \mathrm{m}_{\mathrm{i} 2}^{\mathrm{j}}, \ldots \mathrm{m}_{\mathrm{ij}}^{\mathrm{j}}\right\}
$$

are paths that define a color class of $G$, thus $\varphi_{i}^{k} \cap \varphi_{i}^{j}=\varnothing$

$$
k, j=1,2, \ldots L, k \neq j
$$

and

$$
\bigcup_{j=1}^{L} \varphi_{i}^{j}=V .
$$


When the vehicles of the paths of one phase $\varphi_{i}^{j}$ of the cycle $C_{1}$ then the active pedestrian crossings $P_{k}$ expressed with the natural numbers $\mathrm{k}=1,2, \ldots, \mathrm{np}$ are the elements of the set $[2,3]$

$$
\mathrm{W}_{\mathrm{i}}^{\mathrm{j}}=\left\{\mathrm{k}, /, \varphi_{\mathrm{i}}^{\mathrm{j}} \cap \mathrm{P}_{\mathrm{k}}=\emptyset, \mathrm{k}=1,2, \ldots, \mathrm{np}\right\}
$$

Because each pedestrian crossing must be active in at least one phase of a cycle, the following ratio must be satisfied.

$$
\bigcup_{j=1}^{L} w_{i}^{j}=\bigcup_{j=1}^{L}\{i\}
$$

The creation of light signaling phases that correspond to the $x(G)$ coloring of the in Conflict graph conforms to the above instruction and positively affects the latter.

The concept of multiple colors is related to the third directive.

A cycle is feasible and ultimately acceptable if every pedestrian crossing is active in at least one of its phases, i.e. the relation (2) is valid. The above reasonings have been incorporated into an algorithm developed in the [1] work that creates the $\wp$ family resulting in the SLC (Signal Light Cycles) [1] process. Specifically, two additional routines are applied to each created coloring.

\section{Road Intersection Formulation}

\subsection{Egnatia - Ethnikis Amynis Junction}

The methods and reasoning presented in the previous section were applied at the typical crossroads at the junction of Egnatia and Ethnikis Amynis streets with 11 routes.

Figure 1 shows the relevant intersection as it is today. Table 1 shows the incompatible routes of each route. The corresponding Conflict graph is given in Figure 2.

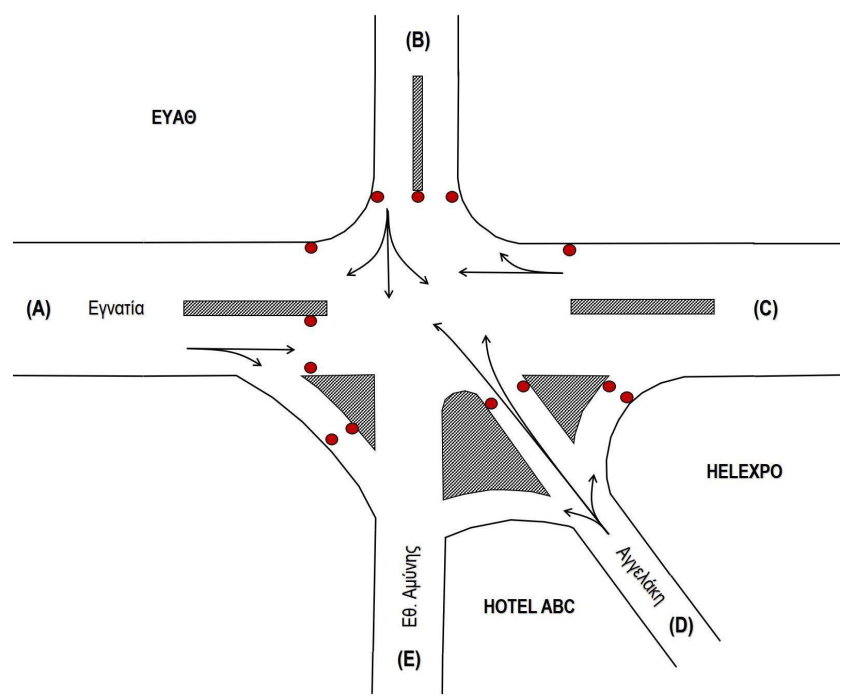

Figure 1. The Egnatia-Ethinkis Amynis junction.

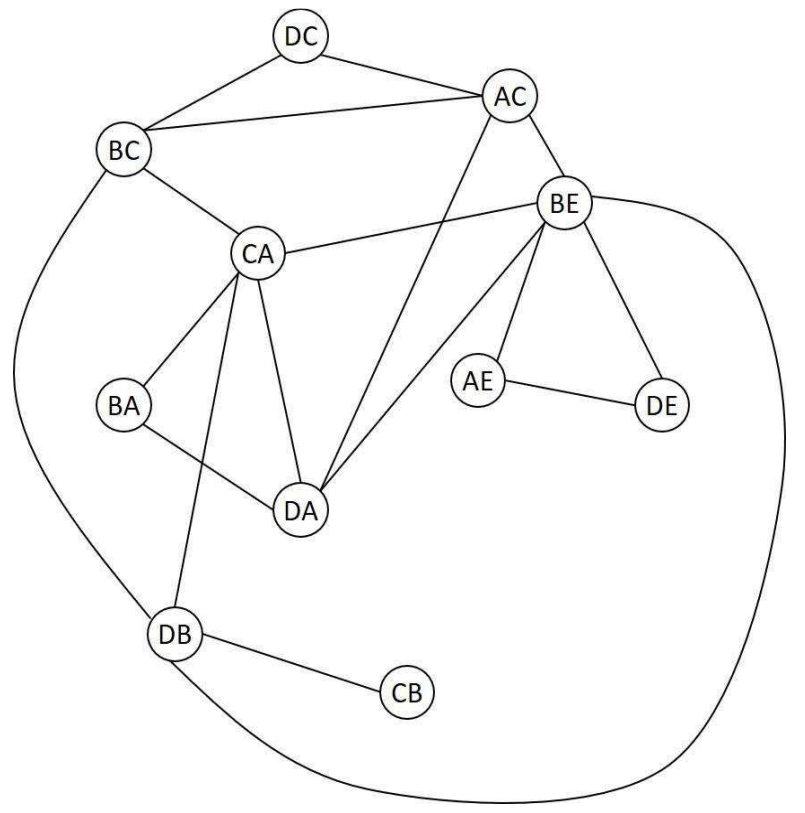

Figure 2. Conflict graph of Figure 1.

Table 1. List array showing vertices of conflict movements.

\begin{tabular}{llll}
\hline Vertices & Conflict movements & Vertices & $\begin{array}{l}\text { Conflict } \\
\text { movements }\end{array}$ \\
\hline BA: & CA DA & CA: & BA BC BE DA DB \\
BE: & DE DA CA AE AC & DC: & AC BC \\
BC: & AC DC CA DB & DB: & CB CA AC BC \\
AC: & BC DC DA DB BE & DA: & CA AC BA BE \\
AE: & BE DE & DE: & BE AE \\
CB: & DB & & \\
\hline
\end{tabular}

Applying the method developed earlier, 4 distinct periods with 3 phases were found. Let us It is recalled that each phase is a color class of the Conflict graph and in the present case its color number is 3 .

Table 2 gives the results for the 4 periods. The subject of the first column is the numbering of the 4 different periods found. The second column contains the main part of the phases of the period. The third column shows the secondary part of the phase.

Table 2. Results of the current state of the junction at Egnatias - Ethnikis Amynis.

\begin{tabular}{lll}
\hline Periods & Main part & Secondary part \\
\hline \multirow{3}{*}{1} & 1. BA BE BCCB & $*$ \\
& 2. AC AE CA & CB \\
& 3. DC DB DA DE & $*$ \\
2 & 1. BA BE BC CB & $*$ \\
& 2. AC CA DE & CB \\
& 3. AE DC DB DA & $*$ \\
3 & 1. BA BE BC & CB \\
& 2. AC AE CB CA & $*$ \\
4 & 3. DC DB DA DE & $*$ \\
& 1. BA BE BC & CB \\
& 2. AC CB CA DE & $*$ \\
& 3. AE DC DB DA & $*$ \\
\hline
\end{tabular}

The $\langle<*>>$ symbol in the secondary part of a phase means that the components of the particular phase constitute a major independent set of the Conflict graph. 


\subsection{The Junction at Egnatias - Ethnikis Amynis with the Aaddition of the AB Route}

In the following section, we present the results of the junction at Egnatias- Ethnikis Amynis with the addition of the $\mathrm{AB}$ route which does not exist until today, thus we have 12 routes in total.

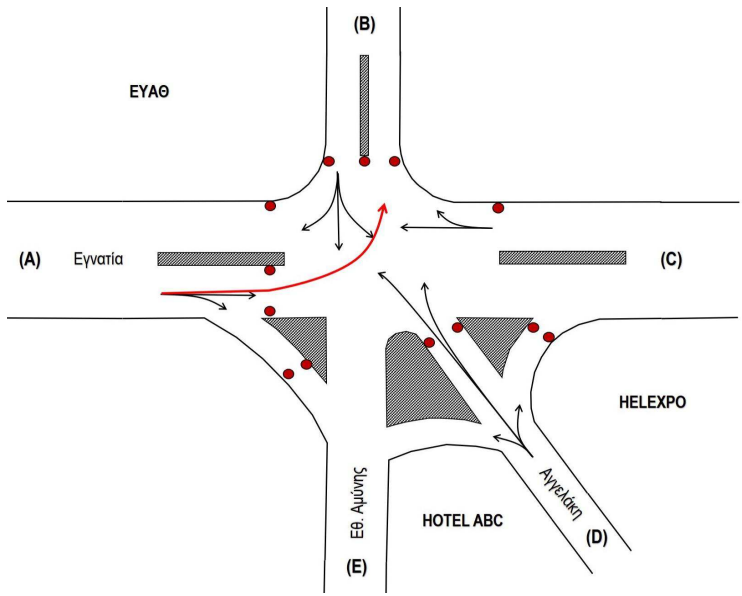

Figure 3. The Egnatia - Ethnikis Amynis junction with the addition of the $A B$ route.
In Figure 3, we see a depiction of the relevant junction to which $\mathrm{AB}$ route was added in red.

In Table 3, we see the incompatible routes of each route. We see the new AB route in bold. The corresponding Conflict graph is given in Figure 4 where we see the new route in red.

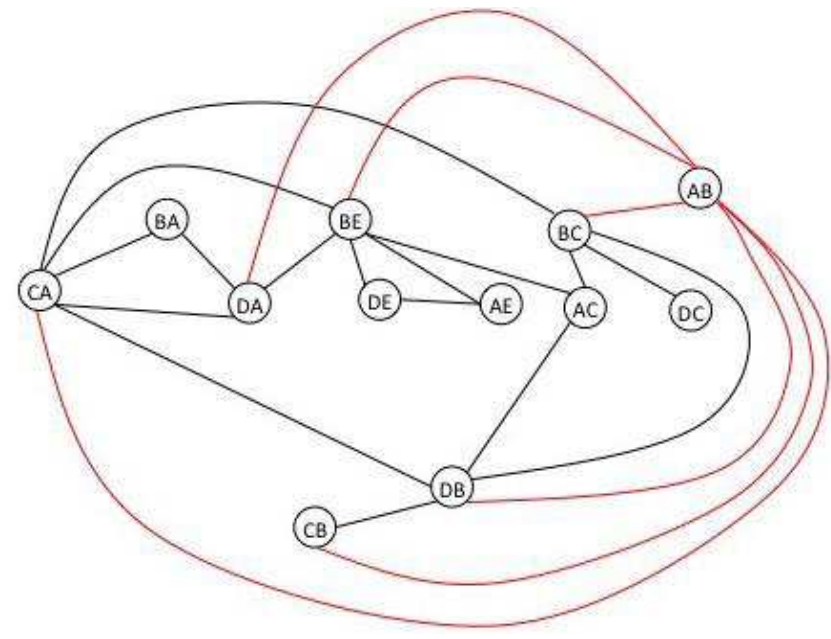

Figure 4. Conflict graph of Figure 3.

Table 3. List array showing vertices of conflict movements.

\begin{tabular}{llll}
\hline Vertices & Conflict movements & Vertices & Conflict movements \\
\hline BA: & CA DA & CA: & BA BC BE DA AB \\
BE: & DE DA CA AE AC & DC: & AC BC \\
BC: & AC DC CA DB AB & DB: & CB CA AC BC \\
AC: & BC DC DA DB BE & DA: & CA AC BA BE \\
AE: & BE DE & DE: & BE AE \\
CB: & DB AB & AB: & CB CA BC BE DB DA \\
\hline
\end{tabular}

Applying the method that was developed earlier and after adding the $\mathrm{AB}$ route, 90 distinct periods were found along with 4 phases. Let's recall that each phase is a color class of the Conflict graph and in this case its color number is 4 .

In Table 4, we see the results for the first phase and the last period respectively. The interpretation for the routes is the same as for the Tables of the previous phases.

Table 4. Results of the Egnatia - Ethnikis Amynis junction with the addition of the $A B$ route.

\begin{tabular}{lll}
\hline Periods & Main part & Secondary part \\
\hline \multirow{4}{*}{1} & 1. BA BE BCCB & $*$ \\
& 2. AC AE CA & CB \\
& 3. DC DB DA DE & $*$ \\
& 4. AB & BA AC AE DC DE \\
90 & 1. BA BE DB & DC \\
& 2. BC DA DE & CB \\
& 3. AC AE AB & BA \\
& 4. CB CA DC & AE DE \\
\hline
\end{tabular}

\subsection{Egnatia - Ethnikis Amynis Junction with the Addition of CE Route}

In this section, we see the results of the Egnatia- Ethnikis Amynis junction with the addition of another route, the $\mathrm{CE}$ route, which does not currently exist and so we have 12 routes in total yet again.
In Figure 5, we see depicted the relevant junction to which $\mathrm{CE}$ route has been added (in red).

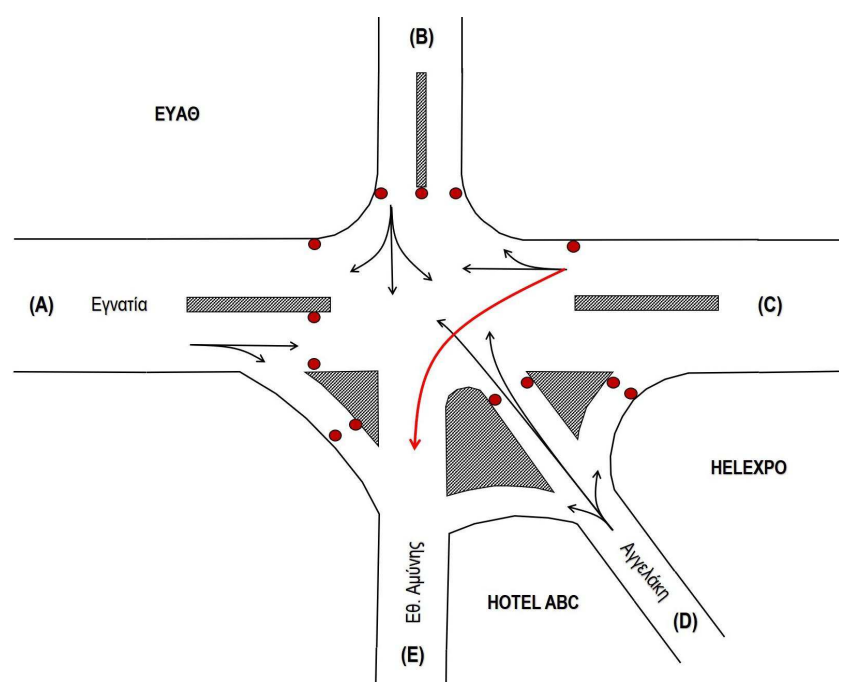

Figure 5. Egnatias - Ethnikis Amynis junction with the addition of CE route.

At Table 5, we see the incompatible routes of each route. The new $\mathrm{CE}$ route is depicted in bold. The corresponding Conflict graph given in Figure 6 where our new route is shown in red. 
Table 5. List array showing vertices of conflict movements.

\begin{tabular}{llll}
\hline Vertices & Conflict movements & Vertices & Conflict movements \\
\hline BA: & CA DA & CA: & BA BC BE DA DB AB \\
BE: & DE DA CA AE AC & DC: & AC BC \\
BC: & AC DC CA DB CE & DB: & CB CA AC BC CE \\
AC: & BC DC CA DB CE & DA: & CA AC BA BE CE \\
AE: & BE DE CE & DE: & BE AE CE \\
CB: & DB AB & CE: & DE DB DA BC BE AC AE \\
\hline
\end{tabular}

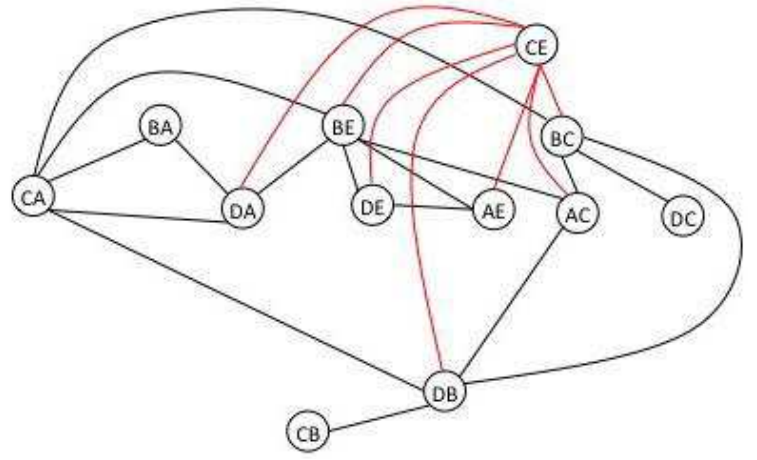

Figure 6. Conflict graph of Figure 5.

Applying the method developed earlier, with the addition of the $\mathrm{CE}$ route, 96 distinct periods with 4 phases were found. It is reminded that each phase is a color class of the Conflict graph and in the present case its color number is 4 .

Table 6 gives some indicative results out of the 96 phases found in total. The interpretation of the routes is the same as for the Tables of the previous sections.

Table 6. Results of the Egnatia - Ethnikis Amynis junction with the addition of the CE route.

\begin{tabular}{lll}
\hline Periods & Main part & Secondary part \\
\hline \multirow{4}{*}{1} & 1. BA BE BCCB & $*$ \\
& 2. AC AE CA & CB \\
& 3. DC DB DA DE & $*$ \\
& 4. CE & BA CB CA DC \\
& 1. BA BE BC & CB \\
24 & 2. AC DE & BA CB CA \\
& 3. AE DB DA & DC \\
& 4. CB CA DC CE & $*$ \\
\hline
\end{tabular}

\subsection{Egnatia - Ethnikis Amynis Junction with the Addition of Both $A B$ \& CE Routes}

The following section presents the results of the Egnatia Ethnikis Amynis junction with the addition of both $\mathrm{AB}$ and $\mathrm{CE}$ routes that do not exist until today and so we have 13 routes in total again.
Figure 7 depicts the relevant crossroads to which the 2 routes $\mathrm{AB}, \mathrm{CE}$ (in red) have been added.

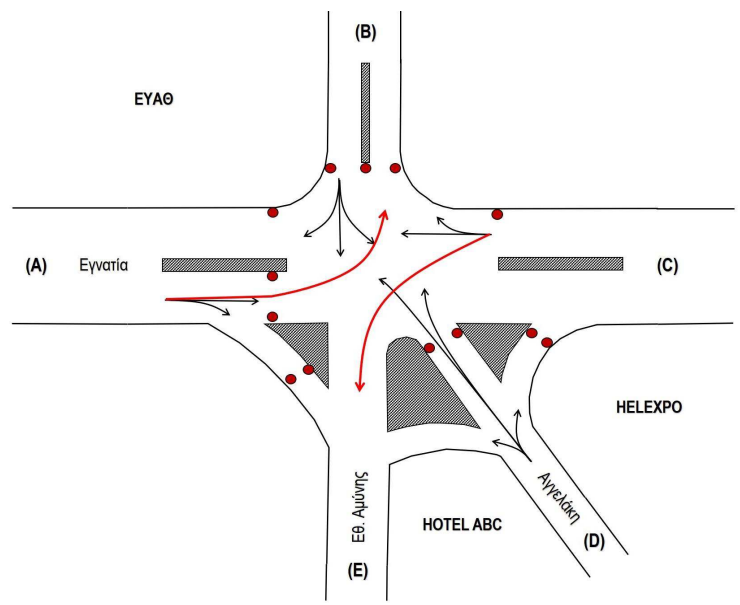

Figure 7. Egnatia - Ethnikis Amynis junction with the addition of both $A B$ \& CE routes.

Table 7 shows the incompatible routes of each route. The new $\mathrm{AB}$ and $\mathrm{CE}$ routes are displayed in bold. The corresponding Conflict graph is given in Figure 8 where our routes are shown in red.

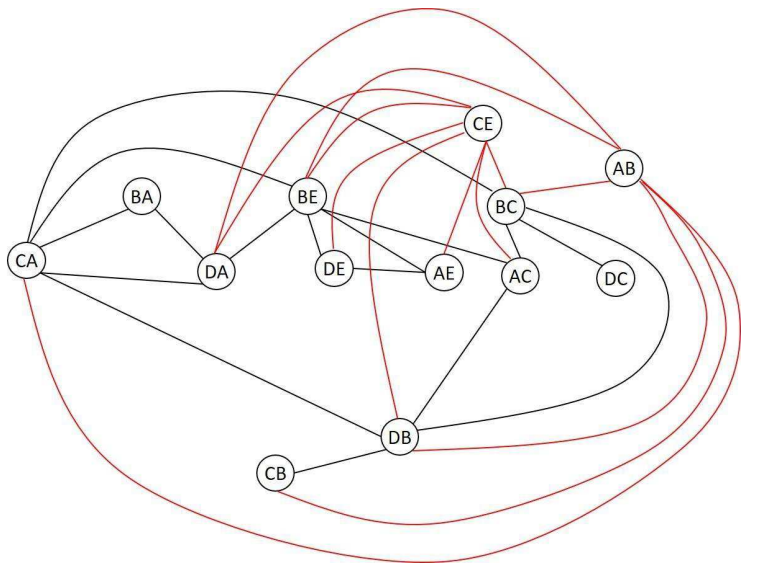

Figure 8. Compatibility graph of Figure 7.

Table 7. List array showing vertices of conflict movements.

\begin{tabular}{llll}
\hline Vertices & Conflict movements & Vertices & Conflict movements \\
\hline BA: & CA DA & CA: & BA BC BE DA DB AB \\
BE: & DE DA CA AE AC & DC: & AC BC \\
BC: & AC DC CA DB CE & DB: & CB CA AC BC CE \\
AC: & BC DC DA DB CE & DA: & CA AC BA BE CE \\
AE: & BE DE CE & DE: & BE AE CE \\
CB: & DB AB & AB: & CB CA BC BE DB DA \\
& & DE DB DA BC BE AC AE \\
\hline
\end{tabular}


Applying the method developed in the previous sections, with the addition of the $\mathrm{AB}$ and $\mathrm{CE}$ routes, 64 distinct periods with 4 phases were found. Please be reminded that each phase is a color class of the Conflict graph $[7,8]$ and in this case its color number is 4 .

Table 8 gives some indicative results out of the 64 phases found in total. The interpretation of the routes is the same as for the Tables of the previous sections.

Table 8. Results Table of the Egnatia - Ethnikis Amynis junction with the addition of the $A B \& C E$ routes.

\begin{tabular}{lll}
\hline Periods & Main part & Secondary part \\
\hline \multirow{4}{*}{1} & 1. BA BE BCCB & $*$ \\
& 2. AC AE CA & CB \\
& 3. DC DB DA DE & $*$ \\
& 4. AB CE & BA DC \\
45 & 1. BA AC DE AB & $*$ \\
& 2. BE DC DB & BA \\
& 3. BC AE CB DA & $*$ \\
64 & 4. CA CE & CB DC \\
& 1. BA AB CE & DC \\
& 2. BE DC DB & BA \\
& 3. BC DA DE & CB \\
\hline
\end{tabular}

\section{Conclusions}

In an urban network and depending on the desired result, the weights assigned to its data refer to cost, time, population size, distances, degree of pollution, landscape coherence, etc.

Graph and network theory are directly related to the management and study of problems related to applications in urban networks:

1. Route \& Coherence problems

2. City spatial problems

Traffic signal design is a complex optimization problem that belongs to the category of NP-Hard problems [9-11]. However, the size of the incompatibility graphs corresponding to road junctions can be solved in acceptable computational time.

The SLC process was applied to a Pavillon 97 Intel (R) notebook, 2.40 Ghz and the corresponding software program needed a small fraction of a sec for the standard Egnatia intersection and less than $40 \mathrm{sec}$ for the multi-way junction presented in the previous sections.

In real life problems the results reported in the set of discrete circles along with the corresponding sub-section and active pedestrian crossings can be stored in electronic files and retrieved every time the route data is changed, so that the duration of the green indicator of each phase is set in real time

Traffic signaling is a dynamic system, so information similar to that mentioned above where all phase cycles are investigated is useful in the process of selecting the most appropriate cycle for the current traffic situation.

\section{References}

[1] Alexiou D., (2018), Generating the family of all traffic signal light cycles coordinated with pedestrian crosswalks, Optimization Letter.

[2] Alexiou D., Katsavounis S. (2013), Determining the Minimum Number of Ware-houses and their Space-Size for Storing Compatible Items, Optimization Theory, Decision Making, and Operations Research Applications, Springer Proceedings in Mathematics \& Statistics 189-198.

[3] Bollobas B. (1998), Modern Graph Theory. Springer-Verlag.

[4] Bondy J. A. (2008), Graph Theory. Springer.

[5] Beineke L. (2004), Robin Wilson and Peter Cameron. Graph theory, Group Theory. Topics in Algebraic Graph Theory Cambridge Univ. Press.

[6] Christofides N. (1975), Graph Theory, an Algorithmic Approach (Academic Press).

[7] Claude B. (1998), Theorie des Graphes et ses Applications, Dunod (Paris).

[8] Neubüser J. An elementary introduction to coset table methods in computational group theory.

[9] Novikov P. S. (1955), On the algorithmic insolvability of the word problem in group theory. Trudy Mat. Inst. Steklov 44, p. $1-143$

[10] Papageorgiou M, Diakaki C., Dinopoulou V., Kotsialos A., and Wang Y (2003)., Review of road traffic control strategies, Proceedings ofthe IEEE, Vol. 91, Issue: 12.

[11] Traffic Signal Operation, Transport Management Center, RTA-TC-106 (2010), http://www.rms.nsw.gov.au/doingbusinesswithus/downloads/te chnicalmanuals/trafficsignalspecifications/rtatc106b.pdf.

[12] Mathew, T., Krishna R.: Traffic signal design-I, Chap. 41, NPTEL (2006).

[13] B. Bollobas: Modern Graph Theory, Springer-Verlag, 1 edition (1998).

[14] Raychaudhuri, A.: Optimal multiple interval assignments in frequency assignment and traffic phasing. Discrete Appl. Math. 40 (3), 319-332 (1992).

[15] Opsut, R. J., Roberts, F. S.: I-Colorings. I-Phasings and Iintersection assignments for graphs, and their applications. Networks 13, 327-345 (1983b). 